\title{
Determinant of Prenatal Diagnostic Testing among Women with Increased Risk of Fetal Aneuploidy and Genetic Disorders
}

\author{
Tamandra Morgan, MD, ScM ${ }^{1}$ Catherine D. Tan, MD $^{2}$ Micaela Della-Torre, MD, MS ${ }^{3}$
} Tia Jackson-Bey, MD, MPH ${ }^{4}$ Laura DiGiovanni, MD $^{3}$ Christopher A. Enakpene, MD

${ }^{1}$ Department of Obstetrics and Gynecology, University of California, San Francisco, San Francisco, California

2 Department of Obstetrics and Gynecology, Advocate Illinois Masonic Medical Center, Chicago, Illinois

${ }^{3}$ Division of Maternal-Fetal Medicine, Department of Obstetrics and Gynecology, Beacon Memorial Hospital, South Bend Indiana

${ }^{4}$ Division of Reproductive Endocrinology and Infertility, Department of Obstetrics and Gynecology, Mount Sinai School of Medicine, New York, New York

${ }^{5}$ Division of Maternal-Fetal Medicine, Department of Obstetrics and Gynecology, School of Medicine, Texas Tech University Health Sciences Center, Permian Basin, Texas

\author{
Address for correspondence Christopher A. Enakpene, MD, Division \\ of Maternal-Fetal Medicine, Department of Obstetrics and \\ Gynecology, School of Medicine, Texas Tech University Health \\ Sciences Center, Permian Basin, 701 West 5th Street, Odessa, TX \\ 79763 (e-mail: cenakpene@gmail.com).
}

Am J Perinatol

\begin{abstract}
Keywords

- genetic counseling

- fetal aneuploidy

- genetic disorders

- diagnostic testing

Objective This study aimed to assess factors that influence patients' decisions in accepting prenatal diagnostic testing following genetic counseling for increased risk of fetal aneuploidy.

Methods This is a retrospective cohort study of women at increased risk of fetal aneuploidy and genetic disorders who had genetic counseling from January 2012 to December 2016 at a single academic center. Demographics, indications for genetic counseling, and rates of diagnostic testing were collected and compared between those who accepted diagnostic testing and those who chose cell free DNA. The variables were analyzed using Chi-square, Fisher's exact test, and multiple logistic regression.

Result Of the 2,373 pregnant women who underwent genetic counseling for increased risk of fetal aneuploidy and genetic disorders during the study period, 321 women had diagnostic testing (13.5\%). Women at 35 years and older accepted diagnostic testing more than women younger than 35 years (20.7 vs. $11.5 \%$, $p<0.001$ ). Asian women accepted diagnostic testing at $27.7 \%$ more than white, non-Hispanic Black, and Hispanic women at 18.0, 12.1, and $11.7 \%$, respectively, $p=0.002$. Number of indications for genetic counseling influenced the likelihood of accepting diagnostic testing. Women with one indication had $11.5 \%$ acceptance of diagnostic testing, and with two and three indications, it was 17.0 and $29.2 \%$,
\end{abstract}

received

January 31, 2021

accepted after revision

October 23, 2021
DOI https://doi.org/

10.1055/a-1692-0309.

ISSN 0735-1631.

\footnotetext{
(C) 2021. The Author(s).

This is an open access article published by Thieme under the terms of the Creative Commons Attribution-NonDerivative-NonCommercial-License, permitting copying and reproduction so long as the original work is given appropriate credit. Contents may not be used for commercial purposes, or adapted, remixed, transformed or built upon. (https://creativecommons.org/ licenses/by-nc-nd/4.0/)

Thieme Medical Publishers, Inc., 333 Seventh Avenue, 18th Floor, New York, NY 10001, USA
} 
respectively. The commonest indication for diagnostic testing was cystic hygroma (risk ratio $[R R]=7.5,95 \%$ confidence interval $[\mathrm{Cl}]: 3.12-8.76 p<0.001$ ). The relative risk of diagnostic testing for fetuses with shortened long bones, femur and humerus, thickened nuchal fold, echogenic bowel, single umbilical artery, and increased nuchal translucency were $4.0,3.3,3.1,2.7$, and 2.7 , respectively. Abnormal serum analyte alone was associated with less acceptance of diagnostic testing $(R R=0.8,95 \% \mathrm{Cl}: 0.7-$ $0.96, p=0.017)$.

Conclusion Age, race, ethnicity, and cumulative number of indications for genetic counseling influenced acceptance of diagnostic testing in at-risk women of fetal aneuploidy and genetic disorders.

\section{Key Points}

- Genetic counseling.

- Fetal aneuploidy.

- Genetic disorders.

- Prenatal diagnostic testing. Prenatal diagnostic testing in women with increased risk of fetal aneuploidy and genetic disorders.

Chromosomal abnormalities are present in approximately 1 in 150 live births. ${ }^{1}$ Congenital malformation is the leading cause of infant death and a leading cause of childhood death. ${ }^{2}$ The United States has one of the highest infant mortality rates among developed nations. In 2013, congenital malformations, deformations, and chromosomal abnormalities accounted for $20.3 \%$ of the total 23,440 infant deaths in the United States with an infant mortality rate of 5.96 per 1,000 live births. ${ }^{3}$ Advances in the application of genomic technology and prenatal ultrasound help to identify approximately 3 to $5 \%$ of pregnancies complicated by chromosomal abnormality, genetic disorders, or birth defects. ${ }^{4}$ First- and second-trimester genetic screening and diagnostic testing affords patients the ability to receive a vast amount of prenatal genetic information about their unborn child. Prenatal invasive or diagnostic tests, such as the chorionic villus sampling (CVS) and amniocentesis, help to analyze fetal genetic information from placental tissue and amniotic fluid, respectively, to ascertain fetal karyotypes and genetic information. ${ }^{5}$

The Society for Maternal-Fetal Medicine and American College of Obstetricians and Gynecologists recommend that all pregnant women including those with abnormal maternal serum markers for fetal aneuploidy or fetal structural abnormality on ultrasound be offered prenatal diagnostic genetic testing using either CVS or amniocentesis with chromosomal microarray analysis. ${ }^{6-8}$ However, current evidence does not support performing diagnostic testing in patients with an isolated ultrasound soft marker for fetal aneuploidy, because soft markers are usually nonspecific and can be transient ultrasound findings, ${ }^{9}$ or in patients with prior negative screening for fetal aneuploidy on first or second trimester screening or cell-free DNA. ${ }^{6}$ Furthermore, the likelihood of fetal chromosomal abnormalities associated with each soft marker varies. The highest likelihood is in fetuses with thickened nuchal fold. ${ }^{10}$ However, the presence of two or more soft markers increases the likelihood of fetal chromosomal abnormalities, and thus warrants consideration for diagnostic testing. ${ }^{11}$ As a recommended clinical practice, all women with increased risk of fetal aneuploidy should be offered referral for genetic counseling. ${ }^{12}$ Genetic counselors educate and counsel patients about genetic disorders through integration of a patient's family and medical histories, and personalized risk assessment of disease occurrence or recurrence. ${ }^{12}$ The decision for patients to undergo diagnostic testing is up to the patient after undergoing genetic counseling. Determinants of the patient decisions have not been well studied.

Previous studies have identified racial and ethnic differences in the utilization of prenatal diagnostic testing in women aged 35 years and older. ${ }^{13,14}$ Some of these differences have been attributed to socioeconomic factors, patient's education, and variations in risk perceptions and receptions. ${ }^{14}$ found variations in decision making for prenatal testing for the Down syndrome among different racial and ethnic groups after accounting for age, marital status, and other socioeconomic factors. ${ }^{15}$ However, few studies with a large number of Black patients explore whether racial and ethnic differences remain since the introduction of cell-free DNA, a noninvasive prenatal screening (NIPS) in 2011. Compared with surrounding academic medical centers in Chicago, IL, the University of Illinois (UIC) Health System treats a diverse patient population, representing mainly racial and ethnic minorities. Currently, there is limited data regarding factors that influence decisions regarding prenatal diagnostic testing among racial and ethnic minority women at increased risk of fetal aneuploidy and genetic disorders. The overall aim of the study is to determine the sociodemographic factors and ultrasound findings that influence a woman's decision to pursue prenatal diagnostic testing following a positive prenatal screen for fetal aneuploidy or genetic disorders. 


\section{Materials and Methods}

This is a retrospective cohort study of pregnant women who had prenatal genetic counseling for increased risk of fetal aneuploidy or genetic disorders from January 1, 2012, to December 31, 2016. They were referred due to a priori of chromosomal or genetic disorders based on personal or family histories or due to abnormal prenatal screening result on serum analytes or first trimester ultrasound abnormalities such as increased nuchal translucency and cystic hygroma or second trimester ultrasound soft markers for fetal aneuploidy. Their medical records were reviewed, and pregnant women with major structural fetal abnormalities on ultrasound, such as congenital cardiac anomaly, genitourinary, gastrointestinal, nervous system, chest, and skeletal anomalies, were excluded from this study. Also excluded from the study were women who had noninvasive prenatal screening (NIPS) based on cell-free fetal DNA as an initial screening test for fetal aneuploidy or if they had prenatal diagnostic testing following abnormal NIPS prior to genetic counseling. During the study period, NIPS was expensive and remained cost-prohibitive for the majority of our patient population, thus its use was restricted as an initial fetal aneuploidy screening except for women who were 35 years or older or who had history of prior pregnancy complicated by fetal aneuploidy, and/or the test was covered by their medical insurance. Those not meeting criteria who did not have medical insurance coverage for NIPS often could only complete the test if they agreed to a self-pay arrangement. All the women received prenatal care at UIC, and they were offered prenatal diagnostic testing with either chorionic villi sampling or amniocentesis for definitive diagnosis during the genetic counseling. They were also offered NIPS using cellfree fetal DNA which became available early in 2012 in the study institution.

\section{Data Collection}

Data were collected with the assistance of the Center for Clinical and Translational Science (CCTS) using the Clinical Research Data Warehouse (CRDW)/UIC CIRCLE as a data source. This was supplemented with a list of patients generated by the research team using diagnostic codes and International Classification of Disease-10th Revision (ICD-10). All eligible patients during the study period were identified using their names, medical record numbers, date of birth, and date of service by the CRDW/UIC; CRDW/UIC/CIRCLE to check for data accuracy. The supplemental list was used to ensure that all eligible patients were included in the study should the CRDW/UIC CIRCLE fail to capture study patients and variables. Research electronic digital capture (REDCap) was used to extract and maintain data. We examined the following indications for genetic counseling: abnormal firstor second-trimesters serum analytes for fetal aneuploidy screening and/or findings on ultrasound such as cystic hygroma, increased nuchal translucency, absent or hypoplastic nasal bone, shortened fetal long bones (femur and humerus), thickened nuchal fold, echogenic bowel, twovessel umbilical cord, and choroid plexus cyst.
Demographic data such as age, race, ethnicity, marital status, medical insurance carrier, religion, and employment status were collected. Additional variables collected were date of genetic screening, date of diagnostic genetic test, chromosomal abnormalities of concerns, indication for and modality of screening for fetal aneuploidy, personal or family history of genetic disorder, combined first- and second-trimester sequential screening, quadruple screening in second trimester, NIPS, and type of prenatal diagnostic testing if performed. All extracted data were entered into the REDCap database and then SPSS for analysis.

\section{Statistical Analysis}

Collected data were analyzed using the IBM SPSS version 24 (IBM Corp, Armonk, NY) software. Comparison of baseline characteristics of the study groups was analyzed. Statistical methods, such as Chi-square and Fisher's exact test were used for categorical variables. Bivariate, multivariate logistic regression analysis was performed to test for covariance and confounding variables. Frequency tables were generated for both baseline characteristics and indications for genetic counseling comparing those who had invasive testing and those who had NIPS.

\section{Results}

Between January 2012 and December 2016, there were 2,373 unique pregnancies that received genetic counseling due to increased risk of fetal aneuploidy or genetic disorders. Diagnostic testing was performed on 321 (13.5\%) pregnancies during the study period. Demographic characteristics of women who received genetic counseling and underwent diagnostic testing were compared with those who declined invasive testing but chose NIPS (-Table 1). Approximately, $20.7 \%$ of women 35 years or older, accepted invasive testing compared with $11.5 \%$ of women less than 35 years old. Hence, older women were more likely to undergo diagnostic testing after undergoing genetic counseling for increased risk of fetal aneuploidy and genetic disorders $(p<0.001)$. In regard to race and ethnicity, Asian women were most likely to proceed with diagnostic testing following genetic counseling. Among the 58 Asian pregnant women who underwent genetic counseling, $27.6 \%$ of them accepted invasive testing compared with $17.8 \%$ of 337 white women, $12.1 \%$ of 969 nonHispanic Black women, and $11.7 \%$ of 300 Hispanic women, respectively, $p=0.002$. The proportion of divorced women who accepted invasive testing (22.7\%) was significantly higher than married or single women, 16.1 and $12.3 \%$, respectively $(p<0.001)$. We also identified differences in acceptance of invasive testing based on the patient's medical insurance and employment status. Pregnant women with Medicare insurance were most likely to accept invasive testing $(20 \%)$, followed by pregnant women with private insurance (17.2\%) and Medicaid (11.6\%), $p=0.005$. Employed women pursued more diagnostic testing at $18.7 \%$ compared with unemployed women at $13.5 \%, p<0.001$. Patients' religious affiliation did not significantly influence their decisions to accept invasive testing or NIPS among women 
Determinant of Prenatal Diagnostic Testing among Women Morgan et al.

\begin{tabular}{|c|c|c|c|}
\hline Variables & Invasive testing $(n=321)$ & Noninvasive testing $(n=2,052)$ & $p$-Value \\
\hline \multicolumn{4}{|l|}{$\begin{array}{l}\text { Age (y) } \\
n(\%)\end{array}$} \\
\hline$<35$ & $214(11.5)$ & $1,642(88.5)$ & \multirow[t]{2}{*}{$<0.001$} \\
\hline$\geq 35$ & $107(20.7)$ & $410(79.3)$ & \\
\hline \multicolumn{4}{|l|}{$\begin{array}{l}\text { Ethnicity/race } \\
n(\%)\end{array}$} \\
\hline African American & $117(12.1)$ & $852(87.9)$ & \multirow[t]{5}{*}{0.002} \\
\hline Hispanic & $35(11.7)$ & $265(88.3)$ & \\
\hline White & $60(18.0)$ & $273(82.0)$ & \\
\hline Asian & $16(27.6)$ & $42(72.4)$ & \\
\hline Other & $93(13.0)$ & $620(87.0)$ & \\
\hline \multicolumn{4}{|l|}{$\begin{array}{l}\text { Marital status } \\
n(\%)\end{array}$} \\
\hline Married & $123(16.1)$ & $640(83.9)$ & \multirow[t]{5}{*}{$<0.001$} \\
\hline Single & $180(12.3)$ & $1,280(87.7)$ & \\
\hline Separated & $2(9.5)$ & $19(90.5)$ & \\
\hline Divorced & $5(22.7)$ & $17(77.3)$ & \\
\hline Other & $11(10.4)$ & $96(89.7)$ & \\
\hline \multicolumn{4}{|l|}{$\begin{array}{l}\text { Medical insurance } \\
n(\%)\end{array}$} \\
\hline Medicare & $5(20.0)$ & $20(80.0)$ & \multirow[t]{5}{*}{0.005} \\
\hline Medicaid & $173(12.0)$ & $1,313(88.0)$ & \\
\hline Private insurance & $137(17.4)$ & $650(82.6)$ & \\
\hline Self-pay & $4(7.5)$ & $49(925)$ & \\
\hline Other & $2(9.1)$ & $20(90.9)$ & \\
\hline \multicolumn{4}{|l|}{$\begin{array}{l}\text { Religion } \\
n(\%)\end{array}$} \\
\hline Baptist & $10(12.1)$ & $73(87.9)$ & \multirow[t]{5}{*}{0.580} \\
\hline Catholic & $13(15.3)$ & $72(84.7)$ & \\
\hline Evangelical & $7(18.4)$ & $31(81.6)$ & \\
\hline Other & 35 (18.6) & $153(81.4)$ & \\
\hline No stated religion & $256(13.0)$ & $1,723(87.0)$ & \\
\hline \multicolumn{4}{|l|}{$\begin{array}{l}\text { Employment status } \\
n(\%)\end{array}$} \\
\hline Employed & $104(18.7)$ & $453(81.3)$ & \multirow[t]{4}{*}{$<0.001$} \\
\hline Unemployed & 199 (13.6) & $1,262(86.4)$ & \\
\hline Students & $8(10.7)$ & 67 (89.3) & \\
\hline Other & $10(3.6)$ & $270(96.4)$ & \\
\hline
\end{tabular}

at increased risk of fetal aneuploidy and genetic disorders who received genetic counseling.

The higher the number of indications for genetic counseling, the more likely invasive testing was performed ( - Table 2 ). Among women who had only one indication for genetic counseling, $11.5 \%$ accepted invasive testing. Approximately $17.0 \%$ of women with two indications for genetic counseling accepted invasive testing while $29.2 \%$ of women with three or more indications accepted invasive testing, $p<0.001$. Patients with an ultrasound finding of cystic hygroma were most likely to accept invasive testing compared with other indications; 52.4\% had prenatal diagnostic testing compared with $47.6 \%$ who declined and underwent NIPS with cell-free DNA. Among other indications for genetic counseling, such as shortened long bones femur and humerus, 38.1\% accepted invasive testing versus $69.1 \%$ who declined. This was followed by patients with ultrasound findings of absent or hypoplastic nasal bone (37.5\%), thickened nuchal fold (33.3\%), echogenic 


\begin{tabular}{|c|c|c|c|}
\hline $\begin{array}{l}\text { Variables } \\
n(\%)\end{array}$ & Invasive testing $(n=321)$ & Noninvasive testing $(n=2,052)$ & $p$-Value \\
\hline \multicolumn{4}{|l|}{ Number of risk factors } \\
\hline One risk factor & $203(11.5)$ & $1,559(88.5)$ & $<0.001$ \\
\hline Two risk factors & $85(16.8)$ & $421(83.2)$ & \\
\hline Three or more risk factors & $33(29.2)$ & $80(70.8)$ & \\
\hline Cystic hygroma & $22(52.4)$ & $20(47.6)$ & $<0.001$ \\
\hline Short femur/humerus & $8(38.1)$ & $13(61.9)$ & $<0.001$ \\
\hline Absent/hypoplastic nasal bone & $3(37.5)$ & $5(62.5)$ & 0.047 \\
\hline Thickened nuchal fold & $11(33.3)$ & $22(66.7)$ & $<0.008$ \\
\hline Echogenic bowel & $13(31.7)$ & $28(68.2)$ & $<0.008$ \\
\hline Two-vessel cord & $10(29.4)$ & $24(70.6)$ & 0.006 \\
\hline Increase NT & $7(29.2)$ & $17(70.8)$ & 0.024 \\
\hline Abnormal analytes & $232(11.2)$ & $1,847(88.8)$ & $<0.017$ \\
\hline Choroid plexus cyst & $15(16.5)$ & $76(88.5)$ & 0.400 \\
\hline
\end{tabular}

\begin{tabular}{|lllll|}
\hline \multicolumn{5}{|l|}{ Table 3 Comparison of indications for invasive testing versus noninvasive testing } \\
\hline $\begin{array}{l}\text { Variables } \\
\boldsymbol{n}(\%)\end{array}$ & Invasive testing $(\boldsymbol{n}=\mathbf{3 2 1})$ & Noninvasive testing $(\boldsymbol{n}=\mathbf{2 , 0 5 2 )}$ & $\mathrm{RR}(\mathbf{9 5 \%} \mathrm{Cl})$ & $p$-Value \\
Cystic hygroma & $22(6.6)$ & $20(1.0)$ & $7.5(4.0-14.0)$ & $<0.001$ \\
Short femur/humerus & $8(2.4)$ & $13(1.0)$ & $4.0(1.6-9.7)$ & $<0.001$ \\
Absent/hypoplastic nasal bone & $3(0.9)$ & $5(0.2)$ & $3.9(0.9-16.3)$ & 0.047 \\
Thickened nuchal fold & $11(3.4)$ & $22(1.1)$ & $3.3(1.6-6.8)$ & $<0.008$ \\
Echogenic bowel & $13(4.1)$ & $28(1.4))$ & $3.1(1.5-5.8)$ & $<0.008$ \\
Two-vessel cord & $10(3.1)$ & $24(1.2)$ & $2.7(1.3-5.7)$ & 0.006 \\
Increase NT & $7(2.2)$ & $17(0.8)$ & $2.7(1.1-6.5)$ & 0.024 \\
Abnormal analytes & $232(72.2)$ & $1,847(90.0)$ & $0.8(0.7-0.96)$ & 0.017 \\
Choroid plexus cyst & $15(4.7)$ & $76(3.7)$ & $1.3(0.7-2.3)$ & 0.400 \\
\hline
\end{tabular}

Abbreviations: $\mathrm{Cl}$, confidence interval; RR, risk ratio; NT, nuchal translucency.

bowel (31.7\%), two-vessel umbilical cord (29.4\%), increased nuchal translucency (29.2\%), abnormal serum analytes (19.5\%), and choroid plexus cyst (16.5\%).

The most statistically significant indication for invasive testing as compared with other indications was the presence of a cystic hygroma on ultrasound (risk ratio $[R R]=7.5,95 \%$ confidence interval [CI]: 4.0-14.0, $p<0.001$; - Table 3). Other indications that significantly influenced whether high-risk women decided to accept invasive testing included shortened long bones femur and humerus $(\mathrm{RR}=4.0,95 \% \mathrm{Cl}: 1.6-9.7$, $p<0.001)$, thickened nuchal fold $(\mathrm{RR}=3.3,95 \% \mathrm{CI}$ : $1.6-$ $6.8,<0.008$ ), echogenic bowel ( $\mathrm{RR}=3.1,95 \% \mathrm{Cl}$ : $1.5-5.8$, $p<0.008$ ), two-vessel umbilical cord (RR 2.7, 95\% CI 1.3-5.7, $p=0.006)$, increased nuchal translucency $(\mathrm{RR}=2.7,95 \% \mathrm{CI}$ : $1.1-6.5, p=0.024)$, and abnormal serum analytes $(\mathrm{RR}=0.8$, 95\% CI: 0.7-0.96, $p<0.017$ ). However, fetuses with absent or hypoplastic nasal bone $(\mathrm{RR}=3.9,95 \% \mathrm{Cl}$ : $0.9-16.3, p=0.05)$, and choroid plexus cyst ( $\mathrm{RR}=1.3,95 \% \mathrm{CI}: 0.7-2.3, p=0.400)$ did not significantly influence a patient's acceptance of prenatal invasive testing. Among all women who had diagnostic testing,
$72.2 \%$ were as a result of abnormal serum analytes which was the highest proportion ( $\mathrm{RR}=0.8,95 \% \mathrm{CI}: 0.7-0.96, p=0.017)$.

\section{Discussion}

\section{Principal Findings}

In our study, we identified that race, marital status, type of medical insurance, and employment status significantly influence patients' decision to undergo prenatal invasive testing when they screened positive for increased risk of fetal aneuploidy or genetic disorders. We found an increased use of invasive testing among divorced women compared with married or single women. Additionally, women who were employed were more likely to pursue diagnostic testing than unemployed women. In our study, religious affiliation did not significantly impact decisions on invasive testing. Our study showed that women with fetuses affected by cystic hygroma were 7.5 times more likely to choose diagnostic testing over NIPS tests than when they had other indications for invasive testing. 


\section{Results}

Previous studies have shown that the acceptance of prenatal invasive testing varies across different racial and ethnic groups. ${ }^{15,16}$ This finding was corroborated by the outcomes of our study which showed that Asian women have the highest rate of acceptance of invasive testing and the rates of acceptance also varied with other races and ethnicity. However, the majority of women in our study that screened positive for fetal aneuploidy still declined prenatal diagnostic testing. The introduction of cell-free DNA further accentuates the racial and ethnic differences in the acceptance of prenatal diagnostic testing. Most participants in this study chose the cell-free DNA over diagnostic testing. The sensitivity of cell-free DNA still depends on the type of fetal aneuploidy being screened for. Cell-free DNA has a sensitivity of more than $99 \%$ for the Down syndrome in pregnancies at high risk for this syndrome, ${ }^{17,18}$ probably without the associated risk of miscarriage from an invasive procedure. ${ }^{18-23}$ Invasive testing is still the definitive diagnosis of fetal aneuploidy, but only 1 in 15 to 20 invasive tests confirms fetal aneuploidy, and the procedure-related pregnancy loss is 1 in 1,000 to 1 in $800 .{ }^{24,25}$ However, a study that compared pregnancy loss in women at increased risk for fetal aneuploidy who underwent immediate diagnostic testing versus cell-fee DNA found that there was no statistically significant difference between the groups. ${ }^{26}$

The presence of a cystic hygroma on ultrasound was the most common indication for invasive testing. A nuchal cystic hygroma is associated with fetal aneuploidy, including Down syndrome, in approximately $50 \%$ of cases. ${ }^{27}$ The higher the number of indications for genetic counseling, the more likely a patient decided to pursue invasive testing suggesting that the combination of multiple individual risk factors, for instance, advanced age, family history, and ultrasound findings had a stronger influence than one risk factor. This finding may be reflective of patients' understanding of perceived risk as either "all or nothing" and a tendency to perceive overall risk as significant if there is a cumulative effect.

\section{Clinical Implication}

In this study, we found that pregnant women with Medicare insurance were most likely to accept diagnostic testing. This finding might suggest that insurance coverage for diagnostic testing increases patient utilization. Therefore, insurance coverage for pregnant patients is essential because it facilitates access to desired services during pregnancy.

Women at high-risk of pregnancy abnormalities may choose to proceed to diagnostic testing for further evaluation. However, in 2007, American College of Obstetricians and Gynecologists (ACOG) recommended that prenatal genetic diagnostic tests should be routinely offered to all pregnant women regardless of age or risk. ${ }^{8}$ Patients choose to undergo diagnostic testing for a variety of reasons. Common indications are advanced maternal age, abnormal first- or second-trimester genetic screening results, known parental carrier status, family history of congenital birth defects, or genetic disorders and maternal preference. $^{28}$ From a patient standpoint, advanced preparation, emotionally, psychologically, and medically may drive the decision to discover offspring diagnosis prior to delivery. For example, ultrasonography can evaluate fetal viability and pregnancy number but it can also evaluate major fetal anomalies in the first trimester like microcephaly, anencephaly, or cystic hygroma. These anomalies may indicate underlined fetal chromosomal disorders, such as Down syndrome and Turner syndrome, or structural abnormalities such as cardiac defects. ${ }^{29}$ Other patients may try to find clinical providers who are experts in certain diseases and make necessary accommodations during the perinatal period. Overall prenatal diagnosis of genetic or structural disorders provides parents the opportunity to receive anticipatory guidance for continuing or terminating an affected pregnancy.

The way patients use this information is dependent on numerous factors. Decisions for prenatal testing may be influenced by region, culture, and society. An international study that recruited pregnant women from Canada, Denmark, Iceland, Israel, Italy, the Netherlands, Portugal, Singapore, and the United Kingdom asked participants about their preferences for prenatal testing and reasons for those choices. The overall majority of women stated that they would select NIPS. However, the majority of women from Portugal and Italy selected diagnostic prenatal testing over NIPS for Down syndrome, and this was contrary to the outcome of our study. One-third of women from the Netherlands and Israel would have opted out of both tests. Hill et al postulates that the larger population of Orthodox Jewish women in Israel may have played a role in their testing decisions due to cultural factors. When asked what factors influenced their decisions, women in Iceland, the Netherlands, and the United Kingdom cited the safety of the tests and procedures as a main contributor to their decision. $^{30}$

Another common reason for proceeding with prenatal diagnostic testing is advanced maternal age, defined as 35 years or older. It is one of the greatest indications for diagnostic testing due to its associated increased risk of chromosomal abnormalities in this demographic. ${ }^{29}$ A study of pregnant Israeli women with normal Down syndrome screening results and normal ultrasound findings, found that being older than 35 years was the most significant factor for pregnant women to ultimately opt for amniocentesis, and this was in stark contrast to previous studies. ${ }^{30,31}$ However, research completed in Taipei found different reasons why patients chose to undergo genetic amniocentesis following positive maternal serum screening. Women in the study cited that amniocentesis was a routine prenatal procedure, they wanted to avoid the risk of having a child with Down syndrome, and they had an inherent trust in technology and their medical providers. ${ }^{32}$ Future areas of research may examine patient decisions about pregnancy outcomes among those who undergo diagnostic testing.

\section{Strengths and Limitations}

The strength of this study was its racial and ethnic diverse study population and location in a large urban medical 
center. This study utilized an existing database which was comprehensive and it contained all the information required for this study. Individual patient's chart was also verified by an independent data manager who was not part of the study's authorship. Limitations of this study include the retrospective cohort design which limits our ability to determine the exact reason why women made certain testing decisions, creating information bias. The low diagnostic testing rate in this study may be related to or a reflection of their insurance coverage. Additionally, we cannot ensure that each patient had the same genetic counseling experience and acknowledge that individual counseling sessions may have also profoundly impacted a patient's ultimate decision about diagnostic testing. Finally, in this study we were unable to assess patient attitudes toward invasive testing before and after genetic counseling to determine what factors went into their decision-making.

\section{Conclusion}

Our study aimed to identify factors that influence a patient's decision about diagnostic prenatal testing after undergoing genetic counseling for increased risk of fetal aneuploidy and genetic disorders. We identified that certain demographic and ultrasound soft markers were more associated with pursuing prenatal diagnostic testing. Provider awareness of this knowledge should influence how we counsel patients and provide them with the necessary tools and resources to make informed decisions that best align with their personal values and goals. The impact of our research would help facilitate genetic counseling, prenatal education, and treatment to be tailored to the individual and population which it serves.

\section{Conflict of Interest}

None declared.

\section{References}

1 Kochanek KD, Kirmeyer SE, Martin JA, Strobino DM, Guyer B. Annual summary of vital statistics: 2009. Pediatrics 2012;129 (02):338-348

2 Osterman MJK, Kochanek KD, MacDorman MF, Strobino DM, Guyer B. Annual summary of vital statistics: 2012-2013. Pediatrics 2015;135(06):1115-1125

3 van Schendel RV, van El CG, Pajkrt E, Henneman L, Cornel MC. Implementing non-invasive prenatal testing for aneuploidy in a national healthcare system: global challenges and national solutions. BMC Health Serv Res 2017;17(01):670

4 Centers for Disease Control and Prevention (CDC) Update on overall prevalence of major birth defects-Atlanta, Georgia, 1978-2005. MMWR Morb Mortal Wkly Rep 2008;57(01):1-5

5 Nussbaum RL, McInnes RR, Willard HF, et al. Thompson \& Thompson Genetics in Medicine. 7th ed. Philadelphia, PA: Saunders/ Elsevier; 2007

6 Norton ME, Biggio JR, Kuller JA, Blackwell SC, Society for Maternal-Fetal Medicine (SMFM). Electronic address: pubs@smfm.org. The role of ultrasound in women who undergo cell-free DNA screening. Am J Obstet Gynecol 2017;216(03):B2-B7

7 Hay SB, Sahoo T, Travis MK, et al. ACOG and SMFM guidelines for prenatal diagnosis: Is karyotyping really sufficient? Prenat Diagn 2018;38(03):184-189
8 ACOG Committee on Practice Bulletins. ACOG practice bulletin no. 77: screening for fetal chromosomal abnormalities. Obstet Gynecol 2007; 109(01):217-227

9 Raniga S, Desai PD, Parikh H. Ultrasonographic soft markers of aneuploidy in second trimester: are we lost? MedGenMed 2006;8 (01):9

10 Nyberg DA, Souter VL, El-Bastawissi A, Young S, Luthhardt F, Luthy DA. Isolated sonographic markers for detection of fetal Down syndrome in the second trimester of pregnancy. J Ultrasound Med 2001;20(10):1053-1063

11 Bromley B, Lieberman E, Shipp TD, Benacerraf BR. The genetic sonogram: a method of risk assessment for Down syndrome in the second trimester. J Ultrasound Med 2002;21(10):1087-1096, quiz 1097-1098

12 Wilson KL, Czerwinski JL, Hoskovec JM, et al. NSGC practice guideline: prenatal screening and diagnostic testing options for chromosome aneuploidy. J Genet Couns 2013;22(01):4-15

13 Kuppermann M, Gates E, Washington AE. Racial-ethnic differences in prenatal diagnostic test use and outcomes: preferences, socioeconomics, or patient knowledge? Obstet Gynecol 1996;87 (5, pt. 1):675-682

14 Kuppermann M, Learman LA, Gates E, et al. Beyond race or ethnicity and socioeconomic status: predictors of prenatal testing for Down syndrome. Obstet Gynecol 2006;107(05):1087-1097

15 Leamen LA, Kuppermann M, Gates E, Nease RF Jr, Gildengorin V, Washington AE. Social and familial context of prenatal genetic testing decisions: are there racial/ethnic differences? Am J Med Genet C Semin Med Genet 2003;119C(01):19-26

16 Saucier JB, Johnston D, Wicklund CA, et al. Racial-ethnic differences in genetic amniocentesis uptake. J Genet Couns 2005;14 (03):189-195

17 Gil MM, Accurti V, Santacruz B, Plana MN, Nicolaides KH. Analysis of cell-free DNA in maternal blood in screening for aneuploidies: updated meta-analysis. Ultrasound Obstet Gynecol 2017;50(03):302-314

18 Norton ME, Jacobsson B, Swamy GK, et al. Cell-free DNA analysis for noninvasive examination of trisomy. N Engl J Med 2015;372 (17):1589-1597

19 Salomon LJ, Alfirevic Z, Audibert F, et al; ISUOG Clinical Standards Committee. ISUOG updated consensus statement on the impact of cfDNA aneuploidy testing on screening policies and prenatal ultrasound practice. Ultrasound Obstet Gynecol 2017;49(06):815-816

20 Committee opinion summary No. 640. Cell-free DNA screening for fetal aneuploidy. Obstet Gynecol 2015;126(03):691-692

21 Society for Maternal-Fetal Medicine (SMFM) Publications Committee. Electronic address: esteele@smfm.org. SMFM Statement: clarification of recommendations regarding cell-free DNA aneuploidy screening. Am J Obstet Gynecol 2015;213(06):753-754

22 Rink BD, Norton ME. Screening for fetal aneuploidy. Semin Perinatol 2016;40(01):35-43

23 Committee on Practice Bulletin- Obstetrics Committee on Genetics Society for Maternal-Fetal Medicine. Practice bulletin No. 163. Screening for fetal aneuploidy. Obstet Gynecol 2016;127(05): e123-e137

24 Simpson JL, Samango-Sprouse C. Prenatal diagnosis and 47,XXY. Am J Med Genet C Semin Med Genet 2013;163C(01):64-70

25 Chasen ST, Skupski DW, McCullough LB, Chervenak FA. Prenatal informed consent for sonogram: the time for first-trimester nuchal translucency has come. J Ultrasound Med 2001;20(11):1147-1152

26 Melan V, Bussieres L, Winer N, et al. Effect of cell-free DNA screening versus direct invasive diagnosis on miscarriage rates in women with pregnancies at high risk of Trisomy 21: a randomized clinical trial. JAMA 2018;320(06):557-565

27 Olivier G, Derniaux A, Alanio E, et al. Characteristics and outcome of fetal cystic hygroma diagnosed in the first trimester. Acta Obstet Gynecolo Scand 2007;86(12):1442-1446

28 Norton ME, Rink BD. Changing indications for invasive testing in an era of improved screening. Semin Perinatol 2016;40(01):56-66 
Determinant of Prenatal Diagnostic Testing among Women Morgan et al.

29 Carlson LM, Vora NL. Prenatal diagnosis: screening and diagnostic tools. Obstet Gynecol Clin North Am 2017;44(02): 245-256

30 Hill M, Johnson JA, Langlois S, et al. Preferences for prenatal tests for Down syndrome: an international comparison of the views of pregnant women and health professionals. Eur J Hum Genet 2016;24(07):968-975
31 Grinshpun-Cohen J, Miron-Shatz T, Ries-Levavi L, Pras E. Factors that affect the decision to undergo amniocentesis in women with normal Down syndrome screening results: it is all about the age. Health Expect 2015;18(06):2306-2317

32 Chiang HH, Chao YM, Yuh YS. Informed choice of pregnant women in prenatal screening tests for Down's syndrome. J Med Ethics 2006;32(05):273-277 